\title{
Efeitos da música na comunicação do idoso: panorama das pesquisas brasileiras
}

Effects of music in communication in elderly: panorama of brazilian researches

Efectos de la música en la comunicación de anciano: panorama de las investigaciónes brasileñas

Lígia Silva PINHEIRO ${ }^{1}$

Andreza Carla Maria da Silva MANSUR ${ }^{2}$

Vanessa Luisa Destro FIDÊNCIO ${ }^{3}$

Luciana Paula MAXIMINO ${ }^{4}$

Camila de Castro CORRÊA ${ }^{5}$

${ }^{1}$ Graduanda em Fonoaudiologia pelo Centro Universitário Planalto do Distrito Federal

${ }^{2}$ Docente do Centro Universitário Planalto do Distrito Federal, Especialista em Disfagia

${ }^{3}$ Docente do Centro Universitário Planalto do Distrito Federal, Doutora em Ciências pela FOB-USP

${ }^{4}$ Livre-docente do curso de Fonoaudiologia, da Faculdade de Odontologia de Bauru - USP

${ }^{5}$ Docente da Universidade de Brasília e do Centro Universitário Planalto do Distrito Federal, Doutora em Bases Gerais da Cirurgia pela FMB-UNESP

\section{Resumo}

Introdução: A fonoterapia pode auxiliar na comunicação de quadros neurológicos, como Acidente Vascular Cerebral, Alzheimer, Parkinson, Esclerose Lateral Amiotrófica e Demências, podendo ser potencializada com a utilização de música associada. Objetivo: Investigar os efeitos da música na comunicação do idoso, baseado nos achados brasileiros. Métodos: A revisão bibliográfica foi estabelecida a partir de buscas realizadas nas bases de dados Google Acadêmico e Portal Regional Virtual Health Library, por meio dos cruzamentos dos seguintes descritores música, idoso e reabilitação. Resultados: As referências pesquisadas expressaram dentre os efeitos na comunicação, a melhora na sequencialização, verbalização, atenção, entonação, prosódia, e a estimulação neuronal, melhorando lateralização, sequência lógica, espaço temporal e memória. Verificou-se também a utilização de métodos como a terapia de entonação melódica, como instrumento de potencialização das habilidades comunicativas comportamentais e consideráveis ativações neuronais. Conclusão: Foi possível verificar que a música traz benefícios nas terapias fonoaudiológicas, em relação à reabilitação na comunicação, canto, fala, linguagem, melhora a prosódia, estimula a memória e a aprendizagem. Sugere-se que futuros estudos busquem padronizar protocolos para o acompanhamento a curto e longo prazo desses possíveis efeitos.

Descritores: Música; Comunicação; Idoso; Fonoaudiologia.

\section{Abstract}

Introduction: Speech-Language Therapy could help the communication of neurological conditions, such as stroke, Alzheimer's, Parkinson's, Amyotrophic Lateral Sclerosis and Dementia, and may be enhanced with the use of associated music. Aim: To investigate the effects of music on elderly communication, in Brazilian researches. Methods: The literature review was established from searches conducted in Google Scholar and Virtual Health Library Portal databases, by crossing the following descriptors Music AND elderly AND rehabilitation. Results: The references researched bring the effects, such as sequencing, verbalization, attention, intonation, prosody, and neuronal stimulation, improving lateralization, logical sequence, temporal space and memory. Methods such as melodic intonation therapy to improve the communicative skills and considerable neuronal activations. Conclusion: It was possible to verify that music brings benefits in Speech-Language Therapy, specifically in rehabilitation in communication, singing, speech, language, improves prosody, stimulates memory and learning. It is suggested that future studies seek to standardize protocols for the short- and long-term follow-up of these possible effects.

Descriptors: Music; Communication; Aged; Language and Hearing Sciences.

\section{Resumen}

Introducción: la terapia del habla puede ayudar en la comunicación de afecciones neurológicas, como derrame cerebral, Alzheimer, Parkinson, esclerosis lateral amiotrófica y demencia, que pueden mejorarse con el uso de música associada, en los datos brasileños. Objetivo: investigar los efectos de la música en la comunicación de las personas mayores. Métodos: La revisión bibliográfica se estableció en base a búsquedas realizadas en las bases de datos Google Scholar y Portal Regional Virtual Health Library, cruzando los siguientes descriptores Música y ancianos y rehabilitación. Resultados: Las referencias investigadas expresaron, entre los efectos sobre la comunicación, la mejora en la secuenciación, verbalización, atención, entonación, prosodia y estimulación neuronal, mejorando la lateralización, secuencia lógica, espacio temporal y memoria. También se utilizaron métodos, como la terapia de entonación melódica, como instrumento para mejorar las habilidades comunicativas conductuales y las activaciones neuronales considerables. Conclusión: fue posible verificar que la música trae beneficios en la terapia del habla, en relación con la rehabilitación en la comunicación, el canto, el habla, el lenguaje, mejora la prosodia, estimula la memoria y el aprendizaje. Se sugiere que los estudios futuros busquen estandarizar protocolos para el monitoreo a corto y largo plazo de estos posibles efectos.

Descriptores: Música; Comunicación; Anciano; Fonoaudiologia.

INTRODUÇÃO

As pesquisas científicas relacionadas à saúde do idoso avançam no Brasil. As mulheres lideram a população idosa com $56 \%$ e os homens com $44 \%$ do grupo etário, como mostram os dados do Instituto Brasileiro de Geografia ${ }^{1}$.

A voz do idoso e as funções corporais sofrem um declínio por volta de 65 anos. $\mathrm{Na}$ senescência, ocorrem alterações naturais fisiológicas e, com o passar dos anos, como consequência, o idoso enfrenta uma série de fatores que desencadeia alterações na qualidade de vida, com diminuição das habilidades em se comunicar, falar, cantar, ouvir, deglutir? .

As habilidades de comunicação são processadas em sítios diferentes. A música, por exemplo, no hemisfério direito, juntamente com - sistema límbico, fornecendo informações emocionais para a fala. A linguagem, no hemisfério esquerdo. Mas a representação musical, como um simples toque de tambor, tem o poder de ativar todas as áreas cerebrais. Pacientes afásicos, em que a lesão está no hemisfério esquerdo, podem ter benefícios com a terapia da entonação (MIT- Melodic Intonation 
Therapy). A dificuldade é na fala, entretanto, é possível realizar entonações melódicas cantar $^{3}$.

Os processos cognitivos musicais no campo da neurociência evoluíram nos últimos anos. A estimulação do campo límbico na maturidade, por meio da música, varia de acordo com cada indivíduo. Estudos da neurociência demostram os efeitos da música no cérebro humano. A música possibilita intervenção dinâmica, favorecendo a saúde no idoso, estimulando melodias entonadas, ritmos variados, escalas musicais, afim de proporcionar 0 equilíbrio da circulação sanguínea, os batimentos cardíacos, harmonizando os efeitos fisiológicos nos cuidados paliativos ${ }^{4,5}$.

A fonoterapia pode auxiliar em desordens da comunicação e deglutição relacionados a quadros neurológicos, como Acidente Vascular Cerebral, Alzheimer, Parkinson, Esclerose Lateral Amiotrófica e Demências $^{5}$. A fluência verbal pode ser desenvolvida por meio de frases musicais, orações ritmadas, prosódia, entonações melódicas $^{6}$. O objetivo deste trabalho foi investigar os efeitos da música na comunicação do idoso, especificamente quanto à estimulação da comunicação, cognição, prosódia, entonação e memória, na literatura científica brasileira.

\section{MATERIAL E MÉTODO}

A busca da revisão daliteratura foi realizada nas interfaces Portal Regional Virtual Health Library (BVS) e Google Acadêmico, com a seguinte estratégia de busca: ("Música" OR "Music" OR "Música") AND ("Idoso" OR "Idosos" OR "Pessoa de Idade" OR "Pessoa Idosa" OR "Pessoas de Idade" OR "Pessoas Idosas" OR "População Idosa" OR "Aged" OR "Anciano" OR "Eldery") AND ("Reabilitação" OR "Rehabilitation" OR "Rehabilitación"). Foram lidos todos os títulos da BVS e do Google Acadêmico foram lidas as 10 primeiras páginas (procedimento de seleção).

\section{- Descrição da estratégia PICO}

A prática baseada em evidência preconiza a utilização de métodos sistemáticos na pesquisa para identificar evidências sobre um determinado tema. Neste sentido, para o presente trabalho, foi utilizada a estratégia PICO (Paciente, Intervenção, Comparação e Outcomes - desfecho) para a construção da pergunta da pesquisa ${ }^{7}$ (Quadro 1).

\section{- Critérios de inclusão e exclusão}

Foram selecionados artigos que tiveram como tema central os efeitos da música como instrumento modificador nas habilidades comunicativas em idosos, como intervenção eficaz. Foram excluídos os artigos que estudaram indivíduos com perda auditiva, que realizaram apenas avaliação, que investigaram outros efeitos (físicos/sistêmicos/psicológicos/sociais) e que não estiveram disponíveis na íntegra pelo Virtual Private Networking (VPN).

Quadro 1. Tabela PICO

\begin{tabular}{|l|l|l|}
\hline Acrônimo & Definição & Especificidade para a presente pesquisa \\
\hline $\mathrm{P}$ & $\begin{array}{l}\text { Paciente ou } \\
\text { problema }\end{array}$ & $\begin{array}{l}\text { Pacientes idoso com alterações na } \\
\text { comunicação }\end{array}$ \\
\hline $\mathrm{I}$ & Intervenção & Música como prevenção e reabilitação \\
\hline $\mathrm{C}$ & $\begin{array}{l}\text { Controle ou } \\
\text { comparação }\end{array}$ & $\begin{array}{l}\text { Intervenção da música na comunicação } \\
\text { idoso }\end{array}$ \\
\hline $\mathrm{O}$ & Outcomes & Benefícios na comunicação com a música \\
\hline
\end{tabular}

- Procedimentos de seleção:

A partir dos cruzamentos, foram lidos os títulos dos artigos localizados para verificar se contemplaram os critérios de inclusão. A partir dos artigos incluídos nesta fase, foram acessados os resumos para segunda análise. Por fim, os resumos incluídos, foram lidos na íntegra, compondo, desta forma, a literatura final para análise dos seguintes itens:

- Autor

- Ano

- Objetivo

- Métodos - casuística

- Métodos - instrumentos de avaliação da comunicação

- Métodos - habilidade da comunicação avaliada

- Métodos - formato da intervenção com música

- Resultados na comunicação por meio da intervenção da música

- Conclusão

RESULTADOS

Por meio das buscas, com o primeiro cruzamento, foram localizados 250 estudos na BVS e lidos 100 estudos no Google Acadêmico, dos quais 13 foram efetivamente incluídos. A seguir, encontra-se a tabela com os respectivos estudos incluídos no presente estudo.

Os temas relacionam-se aos efeitos da música nos processos terapêuticos fonoaudiológicos, estimulando a comunicação, linguagem, fala, verbalização, atenção, entonação, córtex auditivo, memória, espaço temporal, e a utilização de métodos como a Terapia de Entonação Melódica para potencialização das habilidades comunicativas comportamentais e consideráveis ativações neuronais. 
Tabela 1. Resultados das buscas realizadas nas bases de dados Medline, Ibecs, Google Acadêmico

\begin{tabular}{|c|c|}
\hline \multicolumn{2}{|c|}{ Pederiva e Tristão ${ }^{8}$} \\
\hline ANO & 2006 \\
\hline TÍTULO & \begin{tabular}{|l|} 
Música e Cognição \\
\end{tabular} \\
\hline OBJETIVO & Expor os avanços da neurociência cognitiva musical \\
\hline MÉTODOS & $\begin{array}{l}\text { A cognição pode ser desenvolvida por meio da audição por } \\
\text { meio dos códigos musicais. }\end{array}$ \\
\hline RESULTADOS & $\begin{array}{l}\text { Ativação dos órgãos dos sentidos, ampliando a ativação } \\
\text { neuronal }\end{array}$ \\
\hline CONCLUSÂOO & Os avanços da neurociência colaboram para ativação neuronal \\
\hline \multicolumn{2}{|r|}{ קอ } \\
\hline ANO & 2011 \\
\hline TÍTULO & Música e Cognição \\
\hline OBJETIVO & $\begin{array}{l}\text { Compreender como os familiares percebem a influência das } \\
\text { vivências musicais na saúde do familiar que experiencia a } \\
\text { terminalidade }\end{array}$ \\
\hline MÉTODOS & $\begin{array}{l}\text { Sete indivíduos de duas famílias, experenciando a utilização de } \\
\text { música nos cuidados com indivíduos com câncer }\end{array}$ \\
\hline RESULTADOS & Observou-se bem-estar aos pacientes e cuidadores \\
\hline CONCLUSÃO & $\begin{array}{l}\text { A música proporcionou melhor relacionamento interpessoal } \\
\text { entre o doente e sua família }\end{array}$ \\
\hline \multicolumn{2}{|c|}{ Degani e Mercadante $^{10}$} \\
\hline ANO & 2011 \\
\hline TÍTULO & \begin{tabular}{|l} 
Os benefícios da música e do canto na maturidade \\
\end{tabular} \\
\hline OBJETIVO & $\begin{array}{l}\text { Melhorias para a autoestima e auto imagem, destaca-se o } \\
\text { benefício de cantar aumenta as capacidades respiratórias e } \\
\text { pulmonares e a música para auxiliar na reabilitação nos } \\
\text { tratamentos para o idoso }\end{array}$ \\
\hline MÉTODOS & $\begin{array}{l}\text { Estudo de Caso } \\
\text { A música e o canto reabilitam a linguagem e estimulam o } \\
\text { desenvolvimento das habilidades comunicativas ativando as } \\
\text { estruturas subcorticais e o córtex auditivo. }\end{array}$ \\
\hline RESULTADOS & $\begin{array}{l}\text { Importante melhora na comunicação, funções cerebrais, córtex } \\
\text { auditivo, memória e bem estar. }\end{array}$ \\
\hline CONCLUSÃO & $\begin{array}{l}\text { A música e o canto estabelecem estreito relacionamento com a a } \\
\text { linguagem, importante melhora das funções mentais e ativa } \\
\text { áreas cerebrais responsáveis pela função neurológica } \\
\text { relacionadas ás ações motoras, linguísticas e sensoriais }\end{array}$ \\
\hline \multicolumn{2}{|c|}{ Rocha e Boggio ${ }^{11}$} \\
\hline ANO & 2013 \\
\hline TÍTULO & A música por uma óptica neurocientífica \\
\hline OBJETIVO & $\begin{array}{l}\text { A música como intervenção nas alterações neurológicas como } \\
\text { afasia, autismo e dislexia }\end{array}$ \\
\hline MÉTODOS & $\begin{array}{l}\text { A percepção dos sons rítmicos, estimulando as estruturas } \\
\text { cerebrais. Compreensão da importância do estudo da música } \\
\text { com o aumento do corpo caloso. }\end{array}$ \\
\hline RESULTADOS & $\begin{array}{l}\text { Contribuições da música para o tratamento das alterações } \\
\text { neurológicas }\end{array}$ \\
\hline CONCLUSÃO & $\begin{array}{l}\text { A música com relação a linguagem ainda há controvérsias nas } \\
\text { influências causadoras }\end{array}$ \\
\hline \multicolumn{2}{|c|}{ Rodrigues e Caramelli $^{12}$} \\
\hline ANO & 2013 \\
\hline TÍTULO & $\begin{array}{l}\text { Efeitos do treinamento musical no cérebro: aspectos neurais e } \\
\text { cognitivos }\end{array}$ \\
\hline OBJETIVO & $\begin{array}{l}\text { Estudo das evidências realizadas nas últimas duas décadas, } \\
\text { sobre os efeitos dos treinamentos musicais prolongado pode } \\
\text { estar associado a processos neuroplásticos, significantes } \\
\text { mudanças cognitivas }\end{array}$ \\
\hline MÉTODOS & $\begin{array}{l}\text { Revisão das implicações dos resultados dos estudos sobre a } \\
\text { influência da prática musical no cérebro nas áreas de saúde e } \\
\text { educação. }\end{array}$ \\
\hline RESULTADOS & $\begin{array}{l}\text { Os resultados obtidos através do perfil neuropsicológico em } \\
\text { idosos foi possível observar a existências de benefícios capazes } \\
\text { de favorecer a capacidade de reserva cognitiva. }\end{array}$ \\
\hline CONCLUSÃO & $\begin{array}{l}\text { Estudos demonstram os efeitos positivos do treinamento } \\
\text { musical no cérebro, nos aspectos neuronais e cognitivos }\end{array}$ \\
\hline \multicolumn{2}{|c|}{ 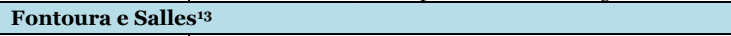 } \\
\hline ANO & 2014 \\
\hline TÍTULO & $\begin{array}{l}\text { Eficácia da terapia de entonação melódica (TEM) aptada: } \\
\text { Estudo de caso de paciente com afasia de Broca }\end{array}$ \\
\hline OBJETIVO & $\begin{array}{l}\text { Testar a eficácia da (TEM), no programa de reabilitação da } \\
\text { linguagem através do canto e música em pacientes com afasia } \\
\text { de Broca }\end{array}$ \\
\hline MÉTODOS & \begin{tabular}{|l|} 
Estudo de caso desenho experimental \\
\end{tabular} \\
\hline RESULTADOS & $\begin{array}{l}\text { Importante melhora na fluência verbal, aumento de números } \\
\text { de palavras produzidas, redução de anomias }\end{array}$ \\
\hline CONCLUSÃO & $\begin{array}{l}\text { A TEM somada a música demostrou eficácia nas terapias para } \\
\text { afasias de Broca. }\end{array}$ \\
\hline \multicolumn{2}{|c|}{ Torres e Freitas 14} \\
\hline ANO & 2015 \\
\hline TÍTULO & $\begin{array}{l}\text { Fonoaudiologia e Musicoterapia na clínica de linguagem: uma } \\
\text { prática clínica }\end{array}$ \\
\hline OBJETIVO & $\begin{array}{l}\text { Estratégias com música em fonoaudiologia para contribuir no } \\
\text { avanço das terapias de linguagem }\end{array}$ \\
\hline MÉTODOS & $\begin{array}{l}\text { Equivalências existentes entre linguagem e comunicação, } \\
\text { relatos de casos na clínica de linguagem }\end{array}$ \\
\hline RESULTADOS & $\begin{array}{l}\text { A conjugação das fundamentações na clínica de linguagem } \\
\text { verbal e ou musical facilita o processo de aprendizagem no } \\
\text { indivíduo. }\end{array}$ \\
\hline CONCLUSÃO & $\begin{array}{l}\text { A música como aliada na expressão fonética, a linguagem } \\
\text { verbal se complementa com a linguagem musical }\end{array}$ \\
\hline \multicolumn{2}{|l|}{ Araújo et al. ${ }^{15}$} \\
\hline ANO & 2016 \\
\hline TÍTULO & $\begin{array}{l}\text { A Musicoterapia no fortalecimento da comunicação entre os } \\
\text { idosos institucionalizados }\end{array}$ \\
\hline OBJETIVO & $\begin{array}{l}\text { Investigar a musicoterapia como promotora da comunicação } \\
\text { entre os idosos de uma determinada Instituição de Longa } \\
\text { Permanência do estado do Piauí }\end{array}$ \\
\hline MÉTODOS & $\begin{array}{l}\text { Método intervencionista para observar informações, construir } \\
\text { um cenário, explorar, analisar e implementar açôes. }\end{array}$ \\
\hline RESULTADOS & $\begin{array}{l}\text { Contribuiu para o fortalecimento da comunicação, propiciando } \\
\text { a sua socialização junto a profissionais e funcionários, além de } \\
\text { consequentes sinais de recuperação da auto-estima. }\end{array}$ \\
\hline $\mathrm{CO}$ & $\begin{array}{l}\text { Ressaltou-se a importância do idoso saber que habilidades e } \\
\text { conhecimentos ainda podem ser adquiridos. }\end{array}$ \\
\hline
\end{tabular}

Tabela 1 (Continuação). Resultados das buscas realizadas nas bases de dados Medline, Ibecs, Google Acadêmico

\begin{tabular}{|c|c|}
\hline \multicolumn{2}{|c|}{ Granja e Carmo $^{16}$} \\
\hline$A N O$ & 2017 \\
\hline TÍTULO & $\begin{array}{l}\text { Música e exercícios fonoaudiológicos para o tratamento de } \\
\text { Doença de Alzheimer (DA) }\end{array}$ \\
\hline OBJETIVO & $\begin{array}{l}\text { Analisar os efeitos da música e exercícios práticos para a } \\
\text { habilitação e reabilitação da linguagem nos Portadores de DA. }\end{array}$ \\
\hline MÉTODOS & $\begin{array}{l}\text { Avaliação por meio do Questionário de Qualidade de Vida } \\
\text { (adaptado), MEEM (mínimo exame do estado mental) } \\
\text { Arizona (grau de Demência), e } 12 \text { sessões de terapias } \\
\text { fonoaudiológicas de 6o'semanalmente }\end{array}$ \\
\hline RESULTADOS & $\begin{array}{l}\text { Melhoria da comunicação } \\
\text { favorecendo a organizçã̃o e planejamento das habilidades de } \\
\text { comunicação e lentificação dos danos cognitivos }\end{array}$ \\
\hline CONCLUSÃO & $\begin{array}{l}\text { A música e Fonoaudiologia contribuíram para a diminuição dos } \\
\text { danos cognitivos }\end{array}$ \\
\hline \multicolumn{2}{|l|}{$\operatorname{Lima}^{17}$} \\
\hline$A N O$ & \begin{tabular}{|l|}
2018 \\
\end{tabular} \\
\hline TÍTULO & $\begin{array}{l}\begin{array}{l}\text { Terapia Entonação Melódica (TEM) para reabilitação da } \\
\text { comunicação na afasia }\end{array} \\
\end{array}$ \\
\hline OBJETIVO & $\begin{array}{l}\text { Avaliar os efeitos da TEM em afásicos, e auxiliar na reabilitação } \\
\text { da comunicação nos transtornos de linguagem }\end{array}$ \\
\hline MÉTODOS & $\begin{array}{l}\text { Intervenção por meio de sessões variadas com música e a a } \\
\text { utilização da TEM, para estimulação da Linguagem em afásicos }\end{array}$ \\
\hline RESULTADOS & $\begin{array}{l}\text { Melhora Linguístico-cognitiva e comportamental nas afasias } \\
\text { emissivas ou receptivas em períodos longos. }\end{array}$ \\
\hline CONCLUSÄO & $\begin{array}{l}\text { A utilização das terapias melódicas beneficia o desempenho de } \\
\text { linguagem cognição e comportamento. }\end{array}$ \\
\hline \multicolumn{2}{|l|}{ Fleming et al. ${ }^{18}$} \\
\hline$A N O$ & \begin{tabular}{|l|}
2019 \\
\end{tabular} \\
\hline TÍTULO & $\begin{array}{l}\text { The effects of short-term musical training on the neural } \\
\text { processing of speech-in-noise in older adults }\end{array}$ \\
\hline OBJETIVO & $\begin{array}{l}\text { Verificar o efeito do treinamento musical curto no desempenho } \\
\text { comportamental }\end{array}$ \\
\hline MÉTODOS & $\begin{array}{l}\text { Participaram idosos não músicos de um treinamento de piano, } \\
\text { videogame e um grupo sem treinamento. Os resultados foram } \\
\text { controlados por ressonância magnética funcional }\end{array}$ \\
\hline RESULTADOS & $\begin{array}{l}\text { Houve melhores respostas corticais à fala nos giros frontais } \\
\text { médios e supramarginais no grupo de treinamento musical }\end{array}$ \\
\hline CONCLUSÃO & $\begin{array}{l}\text { Ressaltaram que o treinamento curto musical em idosos pode } \\
\text { aprimorar a percepção da fala. }\end{array}$ \\
\hline \multicolumn{2}{|l|}{ Lima et al.2o } \\
\hline ANO & 2018 \\
\hline TÍTULO & $\begin{array}{l}\text { Musical and temporal auditory skills in cochlear implant users } \\
\text { after music therapy }\end{array}$ \\
\hline OBJETIVO & $\begin{array}{l}\text { Verificar o desempenho das habilidades de resolução e } \\
\text { ordenação auditiva temporal após musicoterapia em indivíduos } \\
\text { usuários de implante coclear }\end{array}$ \\
\hline MÉTODOS & $\begin{array}{l}11 \text { usuários de IC pós-lingual, submetidos à } 10 \text { sessões } \\
\text { semanais de musicoterapia. } \\
\text { Foi aplicada a Bateria Montreal de Avaliação da Amusia } \\
\text { (MBEA) e do Teste de Padrão de Frequiência (FPT). }\end{array}$ \\
\hline RESULTADOS & $\begin{array}{l}\text { Houve melhora nos subtestes de memória melódica após a } \\
\text { musicoterapia. }\end{array}$ \\
\hline CONCLUSÃA & $\begin{array}{l}\text { A música foi um meio efetivo para abordar as habilidades } \\
\text { auditivas em usuários pós-linguais adultos de Implante } \\
\text { Coclear. }\end{array}$ \\
\hline \multicolumn{2}{|l|}{ Lima et al.2o } \\
\hline ANO & 2018 \\
\hline TÍTULO & $\begin{array}{l}\text { Musical and temporal auditory skills in cochlear implant users } \\
\text { after music therapy }\end{array}$ \\
\hline OBJETIVO & $\begin{array}{l}\text { Verificar o desempenho das habilidades de resolução e } \\
\text { ordenação auditiva temporal após musicoterapia em indivíduos } \\
\text { usuários de implante coclear }\end{array}$ \\
\hline MÉTODOS & $\begin{array}{l}11 \text { usuários de IC pós-lingual, submetidos à } 10 \text { sessões } \\
\text { semanais de musicoterapia. } \\
\begin{array}{l}\text { Foi aplicada a Bateria Montreal de Avaliação da Amusia } \\
\text { (MBEA) e do Teste de Padrão de Frequiência (FPT). }\end{array}\end{array}$ \\
\hline RESULTADOS & $\begin{array}{l}\text { Houve melhora nos subtestes de memória melódica após a } \\
\text { musicoterapia. }\end{array}$ \\
\hline CONCLUSÃO & $\begin{array}{l}\text { A música foi um meio efetivo para abordar as habilidades } \\
\text { auditivas em usuários pós-linguais adultos de Implante } \\
\text { Coclear. }\end{array}$ \\
\hline
\end{tabular}

DISCUSSÃO

O presente estudo revisou temas consideráveis para a fonoaudiologia nos aspectos terapêuticos evolutivos da música no âmbito da melhora na comunicação, fala espontânea, linguagem, cognição e prosódia ${ }^{2-5}$. A tríade fonoaudiologia/ música/ neurociência, fundamenta uma importante abordagem de estimulação nos casos de pacientes acometidos por doenças neurodegenerativas, ou simplesmente para a população adulta e idosa, considerando 0 processo progressivo da senescência natural.

A compreensão sobre a influência da música para o espelhamento neuronal ativo, englobando a comunicação ${ }^{4-6}$, é de suma 
importância para agregar técnicas e estratégias para a terapia fonoaudiológica.

Além disso, a temática se faz relevante quando se trata do idoso, devido ao crescente índice populacional nesta faixa etária, que trará impactos importantes nas possibilidades de atuação, necessitando que a fonoaudiologia amplie sua atuação na gerontologia ${ }^{1}$.

Os achados sobre os impactos da música na terapia fonoaudiológica foram importantes, demonstrando a ampla abrangência destes efeitos, desde habilidades auditivas (de recepção da informação) ${ }^{(20)}$, de memória/cognição ${ }^{12,15-19}$ e até de fala propriamente dita (expressão da linguagem) $(10,13,14)$. Desta forma, a música e o canto são considerados como instrumentos facilitadores, na ativação do corpo caloso, harmonizando os hemisférios cerebrais, estimulando as áreas de Werneck e Broca, auxiliando na comunicação, fala, aprendizagem e cognição. Entretanto, vale ressaltar que a literatura questiona a manutenção desses resultados a longo prazo, mediante a interrupção da estimulação ${ }^{18}$.

Os artigos incluídos nesta revisão datam do período entre os anos 2006 e 2019, demonstrando que o tema se expressa na literatura de modo atual, sendo recentes as investigações que tentam comprovar os efeitos da música na comunicação e cognição do idoso ${ }^{8-17}$.

Em geral, os artigos encontrados estão relacionados com os avanços da neurociência cognitiva musical, terapias de entonação melódicas para afásicos, terapias fonoaudiológicas para disártricos, demonstrando resultados positivos para a respiração, fala, memória, audição, comunicação, cognição, linguagem, entonação e prosódia ${ }^{8-20}$.

Nos estudos selecionados, observou-se a existência de poucos métodos ou protocolos para avaliação da comunicação e não foram coletados dados específicos sobre a quantidade de sessões necessárias para a mensuração dos efeitos da música nas habilidades comunicativas no idoso. Desta forma, sugere-se que futuros estudos busquem padronizar protocolos para o acompanhamento a curto e longo prazo desses possíveis efeitos que a literatura já encontrou.

Com os resultados, os artigos trouxeram contribuições relevantes. A fonoaudiologia, a música e o canto auxiliam na diminuição dos danos relacionados à comunicação. A música e - cantar estabelecem estreito relacionamento com a linguagem ${ }^{6}$, importante melhora das funções mentais e ativa áreas cerebrais responsáveis pelas ações motoras, linguísticas e sensoriais da fala, cognição, entonação, linguagem, comportamento e socialização, memória autobiográfica, qualidade vocal e aprendizagem ${ }^{3,21}$.

Ressalta-se que, apesar de serem achados iniciais, com uma diversidade em relação à métodos (instrumentos), à casuística (quadros de base diversos e número dos participantes), devem ser considerados como fundamentos para o delineamento de novos estudos, que possam comprovar a eficácia da utilização da música na terapia fonoaudiológica. Sugere-se que sejam realizados novos estudos com uma casuística maior, utilização de instrumentos validados para mensuração dos resultados e que o quadro de base do idoso seja considerado por poder interferir nos efeitos diretos da terapia.

CONCLUSÃO

Foi possível verificar que a música traz benefícios para habilidades trabalhadas na terapia fonoaudiológica, em relação à reabilitação na comunicação, canto, fala, linguagem, melhora a prosódia, estimula a memória e a aprendizagem.

\section{REFERÊNCIAS}

1. IBGE (Brasil). Estatísticas Sociais, 24 set. $2018 . \quad$ Disponível em: https://agenciadenoticias.ibge.gov.br/agencianoticias/2012-agencia-de-

noticias/noticias/20980-numero-de-idososcresce-18-em-5-anos-e-ultrapassa-30-milhoesem-2017. Acesso em: 07 fev 2020.

2. Ambrosini E, Cid M, de Isla CG, Salamanca $P$, Borghese NA, Ferrante $S$, et al. Automatic speech analysis to early detect functional cognitive decline in elderly population. Conf Proc IEEE Eng Med Biol Soc. 2019;212-16.

3. Oesch N. Music and Language in Social Interaction: Synchrony, Antiphony, and Functional Origins. Front Psychol. 2019;10:1514.

4. Feast AR, White N, Candy B, Kupeli N, Sampson EL. The effectiveness of interventions to improve the care and management of people with dementia in general hospitals: a systematic review. Int J Geriatr Psychiatry. 2020;35(5): 463-88.

5. Clare A, Camic PM, Crutch SJ, West J, Harding E, Brotherhood E. Using music to develop a multisensory communicative environment for people with late-stage dementia. Gerontologist. 2020;60(6):1115-25.

6. Brotons M, Koger SM. The impact of music therapy on language functioning in dementia. $\mathrm{J}$ Music Ther. 2000;37(3):183-95.

7. Santos CMC, Pimenta CAM, Nobre MRC. A estratégia PICO para a construção da pergunta 
de pesquisa e busca de evidências. Rev. Latino-Am. Enfermagem. 2007;15(3).

8. Pederiva PLM, Tristão RM. Música e Cognição. Ciência E Cognição. 2006;9:83-90.

9. Sales CA, Silva VA, Pilger C, Marcon SS. A música na terminalidade humana: concepções dos familiares. Rev Esc Enferm. USP. 2011; 45(1):138-45.

10. Degani M, Mercadante EF. Os benefícios da música e do canto na maturidade. Kairós Gerontologia. 2010;13(2):149-66.

11. Rocha VC, Boggio OS. A música por uma óptica neurocientífica. Per Musi.2013;27:13240.

12. Rodrigues AC, Loureiro M, Caramelli P: Efeitos do treinamento musical no cerébro: aspectos neurais e cognitivos. Neuropsicologia Latinoamericana. 2013;5(4):15-31.

13. Fontoura DR, Rodrigues JC, Brandão $L$, Monção AM, Salles JF. Eficácia da terapia da entonação melódica adaptada: estudo de caso de paciente com afasia de Broca. Rev Dist Comunic. 2014;26 (4):641-55.

14. Torres LV, Freitas EF. Fonoaudiologia e Musicoterapia na Clínica de Linguagem: uma prática clínica. Estudos Vida e Saúde-EVS. 2015;42(3):45-57.

15. Araújo LF, Santos LMS, Amaral EB, Cardoso ACA, Negreiros F. A Musicoterapia no fortalecimento da comunicação entre os idosos institucionalizados. Rev Kairós. 2016; 19(22,n.esp):191-205.

16. Granja PC, Carmo CF. Música e Exercícios Fonoaudiológicos para tratamento de Alzheimer. Perspectivas Online Biologia e Saúde. 2017;7(23):34-43.

17. Lima GB. Terapia Melódica para Reabilitação da Comunicação na Afasia Revisão Bibliográfica: UNB-DF;2018.

18. Fleming D, Belleville S, Peretz I, West G, Zendel BR. The effects of short-term musical training on the neural processing of speech-innoise in older adults. Brain Cogn. 2019;136: 103592.

19. Fraile E, Bernon D, Rouch I, Pongan E, Tillmann $B$, Lévêque $Y$. The effect of learning an individualized song on autobiographical memory recall in individuals with Alzheimer's disease: A pilot study. J Clin Exp Neuropsychol. 2019;41(7):760-68.

20. Lima JP, lervolino SMS, Schochat E. Musical and temporal auditory skills in cochlear implant users after music therapy. Codas. 2018;30(6): e20180006.

21. Clark IN, Taylor NF, Baker F. Music interventions and physical activity in older adults: a systematic literature review and metaanalysis. J Rehabil Med. 2012;44(9):710-19.

\section{CONFLITO DE INTERESSES}

Os autores declaram não haver conflitos de interesse

\section{AUTOR PARA CORRESPONDÊNCIA}

\section{Camila de Castro Corrêa}

Av. Pau Brasil, lote 01, Águas Claras

71926-000 Brasília-DF, Brasil

Telefone: (61)3435-2200

E-mail: camila.ccorrea@hotmail.com 